\title{
ON THE SOLUTION OF ORDINARY DIFFERENTIAL EQUATIONS OF THE FIRST ORDER INVARIANT UNDER CONTACT TRANSFORMATIONS
}

\author{
MARIO O. GONZÁLEZ
}

In the classical Lie theory it is shown how to construct a differential equation invariant under a given group, and how to solve an equation when a group leaving the equation invariant is known. However, little is said about the problem of determining the group for a given differential equation, which is by far the most interesting problem.

In the present paper, necessary and sufficient conditions for the existence of an infinitesimal contact transformation leaving a given equation invariant are determined along with the general form of the characteristic function of the group. It will also be shown how to reduce, by a proper change of variables, the infinitesimal contact transformation to a point transformation. This enables one to solve the transformed differential equation by Lie's methods. Passing back to the original variables, a new differential equation is obtained which combined with the original equation gives its solution in parametric form.

Let

$$
B f=\xi \frac{\partial f}{\partial x}+\eta \frac{\partial f}{\partial y}+\pi \frac{\partial f}{\partial p}
$$

be the symbol of the infinitesimal contact transformation leaving invariant the differential equation $u=F(v)$, with $u=u(x, y, p)$, $v=v(x, y, p), p=d y / d x$, and $F$ such that the equation $G(x, y, p)$ $=u-F(v)=0$ satisfies the various conditions for the existence of solutions (but otherwise arbitrary). Throughout this paper we shall assume that:

(A) Both $u$ and $v$ have first derivatives with respect to $x, y$ and $p$, at least in some region $R$ of the $(x, y, p)$-space.

(B) The Jacobians

$$
J_{1}=\frac{\partial(u, v)}{\partial(y, p)}, \quad J_{2}=\frac{\partial(u, v)}{\partial(p, x)}, \quad J_{3}=\frac{\partial(u, v)}{\partial(x, y)}
$$

have in $R$ derivatives of the first and second orders, while $J_{1}$ and $J_{2}$ have also derivatives of the third order with respect to $x, y$ and $p$,

Presented to the Society, December 30,1947; received by the editors January 14, 1948, and, in revised form, April 10, 1948. 
as are involved in the discussion.

(C) The functions $u$ and $v$ are not in involution, that is,

$$
[u v]=\left|\begin{array}{cc}
u_{p} & u_{x}+p u_{y} \\
v_{p} & v_{x}+p v_{y}
\end{array}\right|=J_{2}-p J_{1} \neq 0 .
$$

Since $u$ and $v$ are to be invariants under $B f$ they will satisfy the partial differential equations

$$
\begin{aligned}
& \xi \frac{\partial u}{\partial x}+\eta \frac{\partial u}{\partial y}+\pi \frac{\partial u}{\partial p}=0 \\
& \xi \frac{\partial v}{\partial x}+\eta \frac{\partial v}{\partial y}+\pi \frac{\partial v}{\partial p}=0
\end{aligned}
$$

from which we obtain

$$
\frac{\xi}{\partial(u, v) / \partial(y, p)}=\frac{\eta}{\partial(u, v) / \partial(p, x)}=\frac{\pi}{\partial(u, v) / \partial(x, y)}=\sigma,
$$

$\sigma=\sigma(x, y, p)$ being the common ratio. This can be written

$$
\xi=\sigma J_{1}, \quad \eta=\sigma J_{2}, \quad \pi=\sigma J_{3} .
$$

If $W$ is the so-called characteristic function of the infinitesimal contact transformation, we have also

$$
W=p \xi-\eta=\sigma\left(p J_{1}-J_{2}\right) .
$$

Now to find $\sigma$ we recall that ${ }^{1}$

$$
\xi=\frac{\partial W}{\partial p}, \quad \pi=-\frac{\partial W}{\partial x}-p \frac{\partial W}{\partial y} .
$$

As a consequence of (1), (2) and (3) we obtain the system of equations

$$
\left(p J_{1}-J_{2}\right) \frac{\partial \sigma}{\partial p}+\left(p \frac{\partial J_{1}}{\partial p}-\frac{\partial J_{2}}{\partial p}\right) \sigma=0,
$$

(4) $\quad\left(p J_{1}-J_{2}\right) \frac{\partial \sigma}{\partial x}+p\left(p J_{1}-J_{2}\right) \frac{\partial \sigma}{\partial y}$

$$
+\left[\left(p \frac{\partial J_{1}}{\partial x}-\frac{\partial J_{2}}{\partial x}\right)+p\left(p \frac{\partial J_{1}}{\partial y}-\frac{\partial J_{2}}{\partial y}\right)+J_{3}\right] \sigma=0 .
$$

This system may be written in the homogeneous form

${ }^{1}$ See Cohen, An introduction to the Lie theory of one-parameter groups, p. 186. 


$$
\begin{aligned}
& A_{1} f=\frac{\partial f}{\partial p}+M_{1} \frac{\partial f}{\partial \sigma}=0 \\
& A_{2} f=\frac{\partial f}{\partial x}+p \frac{\partial f}{\partial y}+M_{2} \frac{\partial f}{\partial \sigma}=0
\end{aligned}
$$

in which

$$
\begin{aligned}
& \text { (6) } M_{1}=-\sigma \frac{p\left(\partial J_{1} / \partial p\right)-\left(\partial J_{2} / \partial p\right)}{p J_{1}-J_{2}} \\
& \text { (7) } M_{2}=-\sigma \frac{\left(p\left(\partial J_{1} / \partial x\right)-\partial J_{2} / \partial x\right)+p\left(p\left(\partial J_{1} / \partial y\right)-\partial J_{2} / \partial y\right)+J_{3}}{p J_{1}-J_{2}}
\end{aligned}
$$

Adjoining to the system (5) the equations

$$
\begin{aligned}
& \text { (8) } A_{3} f=\left(A_{1} A_{2}\right) f=\frac{\partial f}{\partial y}+\left(A_{1} M_{2}-A_{2} M_{1}\right) \frac{\partial f}{\partial \sigma}=\frac{\partial f}{\partial y}+M_{3} \frac{\partial f}{\partial \sigma}=0 \\
& \text { (9) } A_{4} f=\left(A_{1} A_{3}\right) f=\left(A_{1} M_{3}-A_{3} M_{1}\right) \frac{\partial f}{\partial \sigma}=0 \\
& \text { (10) } A_{5} f=\left(A_{2} A_{3}\right) f=\left(A_{2} M_{3}-A_{3} M_{2}\right) \frac{\partial f}{\partial \sigma}=0
\end{aligned}
$$

we see that the equations

$$
A_{1} M_{3}-A_{3} M_{1}=0, \quad A_{2} M_{3}-A_{3} M_{2}=0,
$$

are necessary and sufficient conditions in order that the system (4) have a solution. The system (5)-(8) implies the Jacobian complete system

$$
\begin{aligned}
& K_{1} f=\frac{\partial f}{\partial x}+\left(M_{2}-p M_{3}\right) \frac{\partial f}{\partial \sigma}=0, \\
& K_{2} f=\frac{\partial f}{\partial y}+M_{3} \frac{\partial f}{\partial \sigma}=0 \\
& K_{3} f=\frac{\partial f}{\partial p}+M_{1} \frac{\partial f}{\partial \sigma}=0 .
\end{aligned}
$$

Either we may solve (12) or the equivalent total differential equation

$$
\left(M_{2}-p M_{3}\right) d x+M_{3} d y+M_{1} d p-d \sigma=0 .
$$

If $f=\psi(x, y, p, \sigma)$ is the solution of (12), then 


$$
\psi(x, y, p, \sigma)=c
$$

will be the solution of (13), and conversely. Equation (14) determines $\sigma$ in terms of $x, y$, and $p$. Since $\sigma$ enters as a factor in $M_{1}$ and $M_{2}$, it is also a factor of $M_{3 .}{ }^{2}$ Hence, equation (13) can be written

$$
d \sigma / \sigma=d \omega(x, y, p)
$$

and so $\sigma$ has the form

$$
\sigma=k e^{\omega(x, y, p)} \text {. }
$$

Several special formulas for $\sigma$ may be found. For instance, if

$$
M_{1}=\phi_{1}(p) \sigma, \quad M_{2}=\phi_{2}(x) \sigma,
$$

then $M_{3}=0$, and equation (13) reduces to

$$
\phi_{2}(x) \sigma d x+\phi_{1}(p) \sigma d p-d \sigma=0,
$$

from which we obtain

$$
\sigma=k \exp \left(\int \phi_{1}(p) d p+\int \phi_{2}(x) d x\right) .
$$

Therefore, the characteristic function takes the form

$$
W=k\left(p J_{1}-J_{2}\right) \exp \left(\int \phi_{1}(p) d p+\int \phi_{2}(x) d x\right)
$$

by virtue of (2). This special case will be of use in some examples to be considered later.

We summarize our results in the following theorem.

THEOREM. The characteristic function $W$ of the infinitesimal contact transformation leaving invariant a given differential equation $u=F(v)$ can be found by the formula

$$
W=k\left(p J_{1}-J_{2}\right) e^{\omega(x, y, p)}
$$

if, and only if, the equations

$$
A_{1} M_{3}-A_{3} M_{1}=0, \quad A_{2} M_{3}-A_{3} M_{2}=0
$$

are both satisfied for all values of $x, y$ and $p$.

Now, to solve the differential equation $u=F(v)$ invariant under the known group

2 If $M_{1}=\sigma N_{1}, M_{2}=\sigma N_{2}$, then $M_{3}=A_{1} M_{2}-A_{2} M_{1}=\sigma\left(\partial N_{2} / \partial p-\partial N_{1} / \partial x-p \partial N_{1} / \partial y\right)$. This relation, together with (11), are the conditions in order that (13) be an exact differential when divided by $\sigma$. 


$$
B f=W_{p} \frac{\partial f}{\partial x}+\left(p W_{p}-W\right) \frac{\partial f}{\partial y}-\left(W_{x}+p W_{y}\right) \frac{\partial f}{\partial p},
$$

we consider two cases:

(A) Both $\xi=W_{p}$ and $\eta=p W_{p}-W$ are free of $p$. This case occurs when $W$ is linear in $p$. Then $B f$ represents an extended point transformation and the equation may be solved by introducing canonical variables.

(B) Either $\xi$ or $\eta$, or both, contain $p$. Then $B f$ represents a general contact transformation.

In this case we may show that by a suitable change of variables the transformation reduces to a point transformation. ${ }^{3}$ To this aim, let us define a finite contact transformation

$$
X=X(x, y, p), \quad Y=Y(x, y, p), \quad P=P(x, y, p)
$$

in the following manner: $X=u, Y \neq X$ in involution with $X$, that is, such that $[X Y]=0$, or

$$
X_{p} \frac{\partial Y}{\partial x}+p X_{p} \frac{\partial Y}{\partial y}-\left(X_{x}+p X_{y}\right) \frac{\partial Y}{\partial p}=0,
$$

and $P$ by the equation $P=Y_{p} / X_{p}$. The symbol for the transformed group will be

(20) $\bar{B} f=\bar{\xi} \frac{\partial f}{\partial X}+\bar{\eta} \frac{\partial f}{\partial Y}+\bar{\pi} \frac{\partial f}{\partial P}=B X \frac{\partial f}{\partial X}+B Y \frac{\partial f}{\partial Y}+B P \frac{\partial f}{\partial P}$.

But $\bar{\xi}=B X=B u=0$ since $u$ is invariant under $B f$. Since $\bar{\xi}=\bar{W}_{P}$ this implies that $\bar{W}$ is free of $P$. Also, we find that $\bar{\eta}$ does not contain $P$ because $\bar{\eta}=P \bar{W}_{P}-\bar{W}=-\bar{W}$. Hence, $\bar{B} f$ is an extension of the point transformation group

$$
U f=-\bar{W}(X, Y) \frac{\partial f}{\partial Y} .
$$

This group can be reduced further by introducing the canonical variables

$$
X^{*}=X, \quad Y^{*}=-\int \frac{\partial Y}{\bar{W}(X, Y)} .
$$

Then the symbol of the infinitesimal transformation assumes the

${ }^{3}$ Cohen, loc. cit. p. 195, proves that the contact transformation reduces to a point transformation by assuming the corresponding differential equation solvable for $p$ in the form $p=\omega(x, y)$. 
simplest form

$$
U^{*} f=\frac{\partial f}{\partial Y^{*}}
$$

The equation $u=F(v)$ when written with the variables $X, Y, P$ takes the form

$$
\phi(X, Y, P)=0 \text {. }
$$

This is also a differential equation, that is, $P=d Y / d X$, since the relation $d Y-P d X=\lambda(d y-p d x)$ which holds for any contact transformation implies $d Y-P d X=0$ whenever $d y-p d x=0$. Since (22) will be invariant under (21) we are in position to solve (22), either directly or by introducing the canonical variables $X^{*}, Y^{*}$ [this last step reduces the equation to the form $\left.d Y^{*} / d X^{*}=G\left(X^{*}\right)\right]$. Let

$$
\psi(X, Y, c)=0
$$

be the solution of (22). Passing back to the original variables we get a second differential equation

$$
\Psi(x, y, p, c)=0
$$

which together with $u=F(v)$ determines the integral curves of the latter in terms of the parameter $p$.

Examples. I. Consider the differential equation

$$
p+y / p=F(x+2 p) \text {. }
$$

Here $u=p+y / p, v=x+2 p$. Hence, it follows that $J_{1}=2 / p, J_{2}=1$ $-y / p^{2}, J_{3}=-1 / p, p J_{1}-J_{2}=1+y / p^{2}, M_{1}=2 \sigma / p, M_{2}=M_{3}=0$.

Formula (16) can be applied with $\phi_{1}(p)=2 / p, \phi_{2}(x)=0$. Therefore, the characteristic function of the group is

$$
W=k\left(1+y / p^{2}\right) p^{2}=k\left(p^{2}+y\right) .
$$

Since a constant factor is irrelevant, we see that equation (24) is invariant under the infinitesimal contact transformation

$$
B f=2 p \frac{\partial f}{\partial x}+\left(p^{2}-y\right) \frac{\partial f}{\partial y}-p \frac{\partial f}{\partial p} .
$$

By taking $X=v=x+2 p$ equation (19) reduces to

$$
2 \frac{\partial Y}{\partial x}+2 p \frac{\partial Y}{\partial y}-\frac{\partial Y}{\partial p}=0 .
$$

The corresponding system of ordinary differential equations is 


$$
\frac{d x}{2}=\frac{d y}{2 p}=\frac{d p}{-1}=\frac{d Y}{0},
$$

from which we obtain $Y=p^{2}+y$ as a particular integral of (25). Finally we have $P=2 p / 2=p$. Introducing the new variables in (24) we get $d Y / Y=d X / F(X)$. Hence, we have

$$
Y=c e^{G(X)}, \quad G(X)=\int \frac{d X}{F(X)} .
$$

Passing back to the variables $x, y, p$ we obtain

$$
p^{2}+y-c e^{G(x+2 p)}=0 .
$$

The system (24)-(26) furnishes the solution of the equation (24).

For instance, if $F(x+2 p)=\tan (x+2 p)$, equations (24) and (26) are respectively

$$
p+y / p=\tan (x+2 p), \quad p^{2}+y=c \sin (x+2 p) .
$$

Solving for $x$ and $y$ we find

$$
\begin{aligned}
& x=-2 t+\arccos (t / c), \\
& y=-t^{2} \pm\left(c^{2}-t^{2}\right)^{1 / 2}
\end{aligned}
$$

which are the parametric equations of the solution, where $t=p$ is the parameter.

II. To apply the method to find the group leaving invariant some familiar types of ordinary differential equations, let us consider first the homogeneous equation

$$
p=F(y / x) \text {. }
$$

We have $u=p, v=y / x, J_{1}=-1 / x, J_{2}=-y / x^{2}, J_{3}=0, p J_{1}-J_{2}$ $=(y-p x) / x^{2}, M_{1}=0, M_{2}=2 \sigma / x, M_{3}=0$. By using formula (16) with $\phi_{1}(p)=0, \phi_{2}(x)=2 / x$, we get (taking $k=-1$ )

$$
W=p x-y \text {. }
$$

Since $W$ is linear in $p$ we obtain the point transformation with symbol

$$
U f=x \frac{\partial f}{\partial x}+y \frac{\partial f}{\partial y},
$$

which corresponds to the so-called homotetic transformation.

For the linear equation $p+P(x) y=F(x)$ we have $u=p+P y$, $v=x, J_{1}=0, J_{2}=1, J_{3}=-P, p J_{1}-J_{2}=-1, M_{1}=0, M_{2}=-\sigma P, M_{3}=0$. 
By putting $\phi_{1}(p)=0, \phi_{2}(x)=-P, k e^{c}=1$ in formula (16) we obtain

$$
W=-\exp \left(-\int P d x\right)
$$

Hence the symbol for the group has the form

$$
U f=\exp \left(-\int P d x\right) \frac{\partial f}{\partial y} .
$$

III. Finally, we shall give a short table of some general types with the corresponding characteristic functions. ${ }^{4}$

Differential Equations

$$
\begin{aligned}
& y=p x+F[x \phi(p)] \\
& y=p x+p F[y \phi(p)] \\
& y+\phi(p)=p F\left[x+\int \phi^{\prime}(p) d p / p\right] \\
& e^{x} \phi(x+y+p)=F\left[e^{x}(p+1)\right] \\
& \frac{y+x \phi(p)}{p+\phi(p)}=x F\left[\log x+\int \frac{\phi^{\prime}(p) d p}{p+\phi(p)}\right]
\end{aligned}
$$

Characteristic Functions$$
k x \phi(p)
$$$$
k y p \phi(p)
$$$$
k[y+\phi(p)]
$$$$
k \phi(x+y+p)
$$$$
k[y+x \phi(p)] \text {. }
$$

HavaNa UNIVERSITY AND

University of Alabama

${ }^{4}$ I am indebted to my former students Miss C. Santana and Dr. R. Peña for the fourth and fifth types shown in the list. 